\title{
RP32
}

\section{Core Characterization and Sample Selection in Heterogeneous Formations}

\author{
M. Sahib (Kuwait Oil Company) \& M. Dernaika*
}

\section{SUMMARY}

Detailed reservoir core characterization is often overlooked in the sampling process for core analysis measurements. Random core sampling is usually performed and the selected plugs are often not associated with proper rock types or the reservoir heterogeneity. This leads to unrepresentative selection of the core samples and raises questions about the effectiveness of the core data in reservoir models and their calibrations.In this study, a robust sampling strategy was followed in reservoir cores from two heterogeneous formations in Kuwait. The objective of the core sampling was to obtain representative plugs for petrophysical and fluid flow analyses that would support dynamic model predictability. The sample selection criteria were based on statistical distribution of litho-types in the core to ensure optimum characterization of the main reservoir units. The litho-types were identified based on porosity and mineralogy variations along core lengths utilizing advanced dual-energy X-ray CT scanning. This technique provided high resolution data at $0.5 \mathrm{~mm}$ spacing to detect all variations in the core. Different porosity cut-offs were assigned for each mineralogy, which resulted in precise identification of the lithotypes in the core. 


\begin{abstract}
Detailed reservoir core characterization is often overlooked in the sampling process for core analysis measurements. Random core sampling is usually performed and the selected plugs are often not associated with proper rock types or the reservoir heterogeneity. This leads to unrepresentative selection of the core samples and raises questions about the effectiveness of the core data in reservoir models and their calibrations.
\end{abstract}

In this study, a robust sampling strategy was followed in reservoir cores from two heterogeneous formations in Kuwait. The objective of the core sampling was to obtain representative plugs for petrophysical and fluid flow analyses that would support dynamic model predictability. The sample selection criteria were based on statistical distribution of litho-types in the core to ensure optimum characterization of the main reservoir units. The litho-types were identified based on porosity and mineralogy variations along core lengths utilizing advanced dual-energy X-ray CT scanning. This technique provided high resolution data at $0.5 \mathrm{~mm}$ spacing to detect all variations in the core. Different porosity cut-offs were assigned for each mineralogy, which resulted in precise identification of the litho-types in the core.

The CT-derived litho-types were compared with wireline density and PEF logs and a good match was observed, adding confidence to the sampling strategy. However, the CT analysis was more efficient in terms of characterization and sample selection because it captured fine variations in the core that were missed in the wireline logs. The statistical analysis of the litho-types yielded volume percentage for each porosity cut-off in the entire core volume, and the plugs were selected to honor the percentage of each identified litho-type and as well as the key reservoir layering .

This statistical analysis of the litho-type distribution in the core, derived from the dual energy X-ray CT scanning, is a new and quick characterization tool that is applied while the core is still in the barrel. This method eliminates sampling bias and allows for each cored litho-type to be equally represented in the plugs acquired for subsequent petrophysical and fluid flow analyses. 